\title{
Estimation of People Movement in Video Based on Optical Flow Block Method and Motion Maps
}

Journal:

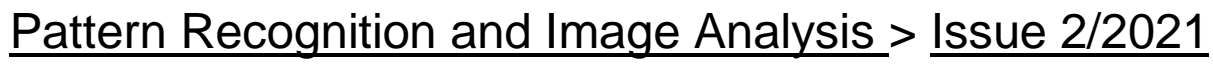

Authors:

H. Chen,

R. P. Bohush,

Ch. Chen,

S. V. Ablameyko

\section{Abstract}

An algorithm for detecting and tracking moving people on video sequences using the block optical flow method and motion maps is proposed. To reduce time expenditures, a pyramidal representation of the frame and template search are used at the stage of building a preliminary map of motion vectors. The integral optical flow allows one to reduce the resulting amplitudes of the background displacement vectors and increase the resulting amplitudes of the displacement vectors of foreground objects. To improve the accuracy for localization of objects, the additive minimax similarity function is used in the analysis of motion vectors. Objects are tracked based on a modified tracing 
algorithm using the Kalman filter. The developed algorithm allows one not only to detect a moving object but also to show the trajectory of its movement. The results of experiments are presented that allow evaluating the effectiveness of the algorithm.

https://www.springerprofessional.de/en/estimation-of-peoplemovement-in-video-based-on-optical-flow-blo/19317748 